\title{
Discussion of "human life is unlimited-but short" by H Rootzén and D Zholud
}

\author{
Olle Nerman ${ }^{1}$
}

Received: 14 June 2018 / Accepted: 18 June 2018 /

Published online: 14 August 2018

(C) The Author(s) 2018

\begin{abstract}
The statistical paper human life is short-but unlimited is briefly discussed. The possibilities and limitations of statistical inference concerning very long human life spans are considered. The restricted models of tail distributions that arise from assumption of renormalized convergence of max- or conditional peaks over thresholdsdistributions are questioned in the application context of the reviewed paper. The restrictions of natural systems designs on possibilities of extreme life spans, and the potential to adopt modified extreme value models, allowing seasonal variation of death rates, are also pointed out.
\end{abstract}

Keywords Long human life lengths · Supercentenarians · Limit of human life spans

Is there a bound for the potential of human life spans? And, if so, can we estimate this bound from empirical data? The first question is not primarily statistical, but rather biological. And, in the era of quick development of organ transplantations, of stem cell therapies and regenerative medicine, and of technical medical spare parts, it is certainly not clear how to generalize life span statistics from the past to predict life spans of the future.

Moreover, on a cellular level it is known that self-renewal is a fundamental part of life, although single cell organisms may survive in dormitory phases (e.g. as seeds or in sporulated forms) for very long times.

For multi-cellular organisms there are controversial indications that stem cells in some circumstances have the potential for indefinite self-renewal, an example is freshwater polyps of the genus Hydra which are thought to have stem cells with this ability and to age at a negligible rate. However, evolutionary theories of ageing that are widely accepted, would claim that the evolution of life spans is governed by a balance

Olle Nerman

nerman@chalmers.se

1 Department of Mathematics, Chalmers University of Technology and University of Gothenburg, Gothenburg, Sweden 
between selective factors that extend the reproductive period and components of intrinsic mortality that shorten it. These do not naturally seem to result in extremely long life spans of complex biological organisms. Few animal species are known to have a potential life span of several hundred years, but humans tend to die with very high probability before the age of 120 years. But theoretically more complex multi-cellular organisms might potentially" be designed" to regenerate indefinitely on the cellular level. An educational style review of the area is available on the Nature link in the end of the reference list. Some other references are (Ackermann et al. 2007), (Kirkwood 1977), (Medawar 1946), (Medawar 1952), (Williams 1957).

As statisticians we typically like to think of a long life span as a combination result of favorable unmeasured inherited factors, systematic and random environmental life style factors, and outcomes of more coincidental random components (possibly with distributions depending on the latter). One might argue that the lives of the very old are more similar between individuals throughout the last years, and that the most important genetic factors of these individuals are less variable than the genetic constitution in the general population. The explanations being strong selection of fit genotypes, and social engagements by their relatives and surrounding communities.

One crucial question in connection with the modelling in the paper we discuss is concerned with the assumption that peaks over threshold distributions in such circumstances can be naturally restricted to generalized Pareto distributions. I do not see any convincing theoretical reasons for such restriction, and as not discussed in the paper, such models might not fit data well for substantially lower thresholds than the one with age 110 that is used.

The main message of the paper, I think, is that one should be careful with modelling of data selected with non-standard bias in the ascertainment step, and the quite remarkable fact that a simple exponential distribution can be reasonably well fitted to explain the 110 years excess life spans of such a heterogenous and large data set as the one studied. The non-ageing interpretation, the empirical observations, and simplicity of the exponential distribution, justify the tentative conclusion concerning the approximate constant death rates in the age interval 110-115, but it does not convince me concerning the death rates in the extrapolation age range of say 120-130 years. Observe also that the approach to handle the selection bias in (Gampe 2010) is similar, and that the exponential distribution conclusion is indicated already in this publication, as properly pointed out by the authors.

I also would like to remark that there probably are quite substantial natural seasonal variations of the death rates of the very old due to seasonality of temperatures, influenza epidemics, etc. It could be interesting to learn more about this through a suitably modified model with seasonality variability embedded. Such modelling naturally requires adaption to regional variability in the timing of seasons and maybe also in effect sizes of those. A general reference of seasonality phenomena in mortality is (Rau 2007).

A few years ago I supervised an undergraduate project in which a student group fitted phase type distributions (absorption times in continuous time finite Markov chains with one absorbing state) to peaks over threshold data. The phase type distribution densities have some parameter- dependent flexibility in their shapes for low values, while all of them have exponential high age tails. The approach is a bit ad hoc, but at least in our examples using cross validations, small dimensional phase type 
models predicted extremes with similar accuracy as fitted generalized Pareto distributions. Perhaps it could be of some interest to retest the null hypothesis of an exponential distribution in various subclasses of phase type distributions (e.g. Coxian distributions) to get additional insights. A useful survey of phase type distributions is (Bladt 2005) and there is also a resent textbook (Bladt and Nielsen 2017).

I also think that one should try an empirical likelihood modification of the authors' approach with discrete peaks over threshold distribution having all probability masses in the overshoots of actually observed life spans in order to derive a nonparametric survival distribution. This could then be, at least graphically, compared to the survival distribution of the fitted exponential. Ideally this requires life spans measured on at least monthly precision level, which might be a problem. I think that this should result in a natural refinement of the analysis in (Gampe 2010).

Acknowledgements I wish to thank my colleague Docent Marija Cvijovic for valuable discussion and help with the biological references below.

Open Access This article is distributed under the terms of the Creative Commons Attribution 4.0 International License (http://creativecommons.org/licenses/by/4.0/), which permits unrestricted use, distribution, and reproduction in any medium, provided you give appropriate credit to the original author(s) and the source, provide a link to the Creative Commons license, and indicate if changes were made.

\section{References}

Ackermann, M., Chao, L., Bergstrom, C.T., Doebeli, M.: On the evolutionary origin of aging. Aging Cell. 6, 235-244 (2007)

Bladt, M.: A review on phase-type distributions and their use in risk theory. ASTIN Bulletin. 35(1), 145-161 (2005)

Bladt, M., Nielsen, B.F. Matrix-Exponential Distributions in Applied Probability. Probability Theory and Stochastic Modelling, vol 81. Springer, Boston, MA (2017)

Gampe, J.: Human mortality beyond age 110. In: Maier, H., Gampe, J., Jeune, B., Robine, J.-M., Vaupel, J.W. (eds.) Supercentenarians, pp. 219-230. Springer, Berlin (2010)

Kirkwood, T.B.L.: Evolution of ageing. Nature. 270, 301-304 (1977)

Medawar, P.B.: Old age and natural death. Modern Quarterly. 1, 30-56 (1946)

Medawar, P.B.: An Unsolved Problem of Biology. Inaugural lecture, London, UK: H. K. In: Lewis and Co (1952)

Rau, R.: Seasonality in Human Mortality. A Demographic Approach. Demographic Research Monographs Series. Springer, Heidelberg (2007)

Williams, G.C.: Pleiotropy, natural selection, and the evolution of senescence. Evolution. 11, 398-411 (1957). https://www.nature.com/scitable/knowledge/library/the-evolution-of-aging-23651151 